\title{
Silent brain infarction after minimally invasive cardiac surgery with retrograde perfusion
}

\author{
Shuhei Nishijima ${ }^{1}$, Yoshitsugu Nakamura ${ }^{1}$, Daiki Yoshiyama ${ }^{2}$, Yuto Yasumoto ${ }^{2}$, Miho \\ Kuroda $^{1}$, Taisuke Nakayama ${ }^{1}$, Ryo Tsuruta ${ }^{1}$, Yujiro Ito ${ }^{1}$, Fumiaki Shikata ${ }^{2}$, Tetuji \\ Takeda $^{2}$, and Nobuyuki Kato ${ }^{2}$ \\ ${ }^{1}$ Chibanishi General Hospital \\ ${ }^{2}$ Affiliation not available
}

May 18, 2020

\begin{abstract}
Background and aim: The incidence of symptomatic cerebral infarction after minimally invasive cardiac surgery (MICS) with retrograde perfusion has been increasing. However, there is no report about silent brain infarction (SBI) after MICS with retrograde perfusion. Because SBI may cause delirium and decline of cognitive function, this condition is important clinically. Thus, the current study aimed to investigate the occurrence of SBI after MICS via magnetic resonance imaging (MRI). Methods: Between July 2014 and July 2018, 174 patients underwent MICS with retrograde perfusion and postoperative MRI in this study. Univariate and multivariate analyses were performed to examine the occurrence of SBI and to identify its risk factors. Results: Of 174 patients, $26(14.9 \%)$ presented with SBI. The univariate analysis revealed that age and aortic valve stenosis (AS) are the risk factors of SBI. Meanwhile, multivariate analysis revealed AS as the only risk factor of SBI. Conclusions: At our institution, the incidence of SBI after MICS with retrograde perfusion was acceptable.
\end{abstract}

\section{Introduction}

The incidence of symptomatic cerebral infarction after minimally invasive cardiac surgery (MICS) was higher than that after median sternotomy because of the retrograde blood flow from the femoral artery ${ }^{1}$. However, as MICS techniques are more widely used, the incidence of symptomatic brain infarction due to retrograde perfusion via the femoral artery has decreased. Recent studies have shown that the incidence was similar to that after median sternotomy ${ }^{2}$. In Japan, the incidence rate of stroke after MICS is reported as $1.0 \%^{3}$. At our institution, the incidence of symptomatic cerebral infarction after MICS was zero ${ }^{4}$.

The significance of symptomatic cerebral infarction after cardiac surgery has been discussed in some studies, and some reports showed that silent brain infarction (SBI) also poses a risk of postoperative delirium ${ }^{5}$. In addition, some studies have indicated that it may accelerate the development of vascular dementia ${ }^{6}$, which is an important clinical factor that can affect quality of life. The incidence rates of SBI are $29 \%$ after median sternotomy ${ }^{5}$ and $29 \%$ after coronary angiography and stenting ${ }^{7}$. This result indicates that the incidence of SBI is not low. However, no previous report has provided detailed information about SBI after MICS with retrograde perfusion via femoral cannulation.

In this study, the detailed characteristics of SBI after MICS were evaluated on head magnetic resonance imaging (MRI).

Materials and Methods

Patient selection and data collection 
After obtaining approval from the review board of Chiba Nishi General Hospital, we performed a prospective, observational study of data collected from patients who underwent right mini-thoracotomy, which is a minimally invasive surgery, at Chiba Nishi General Hospital between July 2014 and July 2018. All patients were diagnosed based on cardiac catheterization and echocardiography findings. During the study period, there were 273 patients who underwent MICS and MRI, including diffusion-weighted imaging (DWI) sequences, the day before and 5 days after surgery. We selected the femoral artery for retrograde perfusion and the axillary artery or ascending aorta for antegrade perfusion. Preoperative computed tomography (CT) angiography was routinely performed, and vascular pathology was evaluated.

The femoral artery was chosen as the cannulation site if the patients met the following criteria: 1) no calcification in the entire circumference of the aorta, 2) thrombosis in less than one-third of the aorta, and 3) thrombosis in the aorta with thickness $<3 \mathrm{~mm}$. In patients who did not meet the criteria, antegrade cannulation was performed.

Of 273 patients, $175(64.8 \%)$ underwent femoral cannulation for CPB and 95 (34.4\%) axillary arterial cannulation. Moreover, one (0.37\%) patient had both femoral cannulations, 1 patient $(0.37 \%)$ femoral and axillary arterial cannulations, and remaining 1 patient $(0.37 \%)$ ascending aorta cannulation. Patients who underwent axillary arterial cannulation, both femoral and axillary arterial cannulations, and ascending aorta cannulation were excluded. Moreover, one patient who presented with a subdural hematoma 3 days after the surgery was excluded. Finally, 174 patients were included in the study (Figure 1).

Patients who were scheduled for right mini-thoracotomy underwent MRI, including DWI sequences, the day before and 5 days after surgery. SBIs detected via DWI were categorized as follows: A) 1-3 DWI spots measuring $<10 \mathrm{~mm}, \mathrm{~B})>3$ DWI spots measuring $<10 \mathrm{~mm}$, and C) single DWI lesion measuring $>10 \mathrm{~mm}$ (Figure 2).

A high field strength (3T) MRI unit was used. The protocol included axial T2-weighted imaging, axial T2-weighted fluid attenuation inversion recovery imaging, axial trace-weighted DWI, and apparent diffusion coefficient mapping.

All images were evaluated by a diagnostic radiologist. When lesions were detected, neurosurgeons were consulted. Renal failure was defined as the requirement of hemodialysis or an elevated creatinine level at 2.0 $\mathrm{mg} / \mathrm{dL}$, which is two times the preoperative baseline level. Thirty-day mortality was defined as all deaths within 30 days after surgery regardless of where the patients died (in- or out-of-hospital).

\section{Use of anesthetics, surgical technique, and postoperative management}

MICS was performed via a 5-6-cm lateral right thoracotomy. The surgical approaches used were via the third intercostal space in aortic valve surgery and via the fourth intercostal space in mitral valve surgery.

In case of retrograde perfusion, we cannulated the femoral artery via a small skin incision in the inguinal region with the direct cannulation technique. The femoral vein was cannulated with a venous cannula, and transesophageal echocardiography was performed to assess the guide wire and cannula in the bicaval view.

Cross-clamping was performed directly via the incision. In both the aortic and mitral valves, antegrade cardioplegia was delivered via a root cannula into the aortic root.

The removal of air was performed via a vent placed in the aortic root and left ventricle in aortic valve surgery and via a vent in the aortic root in mitral valve surgery.

In our hospital, $\mathrm{CPB}$ is initiated with a perfusion index of $2.6 \mathrm{~L} / \mathrm{min} \times \mathrm{m}^{2}$ and conducted with a systemic temperature of $32^{\circ} \mathrm{C}$.

The procedures were performed under direct visualization with thoracoscopic guidance. $\mathrm{CO}_{2}$ insufflation into the right thoracic cavity was performed with a flow rate of $5 \mathrm{~L} / \mathrm{min}$ during the procedure.

\section{Statistical analysis}


Continuous data were presented as mean and standard deviation within the normal range. These data were analyzed using student's and independent sample $t$-test or Mann-Whitney U test. Meanwhile, categorical variables were expressed as counts and percentages, and they were analyzed using chi-square test or Fisher's exact test. Univariate and multivariate logistic regression analyses were conducted to determine the risk factors for SCI after MICS. A P value [?]0.05 was considered statistically significant. Statistical analysis was conducted using the Statistical Package for the Social Sciences software version 25.0 (IBM, NY).

\section{Results}

\section{Patient characteristics and operative data}

The mean age of the patients was 65.0+-14.2 years, and $92(52.9 \%)$ men were included in the study. Moreover, six $(4.0 \%)$ patients had a history of stroke (Table 1$)$.

The procedures comprised single valve surgeries $(n=143,81.3 \%)$, double valve surgeries $(n=20,11.5 \%)$, and other cardiac surgeries $(\mathrm{n}=11,6.3 \%)$. Single valve surgeries comprised aortic valve replacements $(\mathrm{n}=66$, $37.9 \%)$ and mitral valve procedures $(\mathrm{n}=77,44.2 \%)$. The mitral valve procedures included mitral valve repairs $(\mathrm{n}=69,39.6 \%)$ and mitral valve replacements $(\mathrm{n}=8,4.6 \%)$. The double valve procedures comprised aortic and mitral valve surgeries $(\mathrm{n}=4,2.3 \%)$ and mitral and tricuspid valve surgeries $(\mathrm{n}=16,9.2 \%)$.

The median times taken to perform aortic cross-clamping and CPB were 102.9+-37.5 and 132.7+-38.6 min, respectively (Table 2).

\section{Outcomes}

The postoperative outcomes are shown in Table 3. There was no mortality, and none of the patients had stroke (Table 3). In total, $26(14.9 \%)$ patients were diagnosed with SBI based on the postoperative MRI findings. The incidence of SBI after each surgery is shown in Table 4. Two (7.7\%) patients had a history of stroke. The patients were categorized according to severity: A) 1-3 DWI spots measuring $<10 \mathrm{~mm}(\mathrm{n}=22)$ and B) $>3$ DWI spots measuring $<10 \mathrm{~mm}(\mathrm{n}=4)$.

The total number of SBI lesions in 26 patients was 61 . Based on the shape and size of the lesion, the etiology of SBI was interpreted as embolism due to microdebris and air by MRI image reading. The anatomical location and number of SBIs are summarized in Table 5. The location of ischemic foci was divided into cerebrovascular territories, including watershed regions. In total, 34 (56\%) SBI lesions were in the right hemisphere and 27 (44\%) in the left hemisphere. Most SBIs were observed in the posterior cerebral artery territory.

In the univariate analysis, the patients with SBI were older than those without SBI $(\mathrm{P}<0.05)$, and the number of patients with AS was higher than that of patients without SBI $(\mathrm{P}<0.05$; Table 1$)$. Meanwhile, the multivariate analysis revealed that the risk factor for postoperative SBI was aortic valve replacement for aortic stenosis (odds ratio: 2.668, 95\% confidence interval: 1.054-6.754, $\mathrm{P}=0.038$; Table 6). There was no recorded death among the patients diagnosed with SBI who were discharged from the hospital.

\section{Discussion}

\section{Clinical significance of SBI}

To identify the clinical significance of SBI, Knipp et al. evaluated new-onset SBI and postoperative cerebral function after on-pump CABG surgeries by MRI. Moreover, they reported that in the group with SBI, cognitive deterioration was observed during the acute postoperative period compared to the group without $\mathrm{SBI}{ }^{8}$. Sun et al. showed that postoperative delirium was associated with $\mathrm{SBI}^{5}$. This condition can occur when SBI occurs in the frontal lobe or in areas correlated to cortical speech or mental activity. Vermeer et al. ${ }^{9}$ revealed that the risk of dementia increased with spontaneous SBI, and the decline in cognitive function was steep. The occurrence of SBI after cardiac surgery may also affect long-term life expectancy and healthy life expectancy. In the current study postoperative delirium and the cognitive function was not quantified. We believe further research and follow-up are required for patients with postoperative SBI. 


\section{Incidence of SBI}

The incidence rate of SBI was 14.9\% in patients who underwent MICS with retrograde perfusion. According numbers of previous study, SBI is common in cardiac surgeries or catheterization ${ }^{10}$. Compared to previous reports about SBI after median sternotomy, the incidence of SBI in this study was low. In the study of Sun et al., the incidence rate of SBI after median sternotomy was $29 \%$ (5). A detailed review of this report revealed that the incidence rates were $45 \%$ after on-pump coronary artery bypass grafting (CABG) ${ }^{8}, 47 \%$ after valve surgery ${ }^{11}, 31 \%$ after off-pump $\mathrm{CABG}^{12}, 22 \%$ after catheterization of aortic valves ${ }^{13}, 10.2 \%$ after coronary angiography ${ }^{14}, 29 \%$ after coronary angiography and percutaneous coronary intervention ${ }^{7}$, and $34.7 \%$ after urgent percutaneous coronary intervention ${ }^{15}$.

The two possible causes for the low incidence of SBI in our institution were as follows: First, our criteria for femoral cannulation might have affected the incidence of SBI. At our institution, all patients underwent whole-body contrast-enhanced CT scan before surgery, and vascular properties were evaluated using our criteria. Because retrograde perfusion is not used in patients with poor vascular properties, only those with comparatively good vascular properties were included in this analysis. The second cause for the low incidence of SBI is that the thoracic cavity was filled with $\mathrm{CO}_{2}$ at the time of surgery. However, measures were taken to ensure that air does not enter the blood, and air removal was performed using left atrioventricular vent and route vent. Thus, the effect of air embolism was reduced.

\section{Location of SBI}

Based on the evaluation of SBI location, a high number of lesions were localized in the right hemisphere. We speculated the possible causes of SBI in the right hemisphere, which were as follows: First, MICS is performed while the patients are in the left lateral decubitus position at our institution, and this might have made it easier for debris and air to reach the right-dominant vasculature area. Floyed et al. reported a similar tendency on MRI after median sternotomy ${ }^{6}$. In the reports of Knipp et al. ${ }^{11}$ and Barbut ${ }^{16}$, the percentage of embolic lesions in the posterior cerebral circulation was high. However, the actual cause of lesion localization in these studies was not identified. Second, the brachiocephalic artery, which is the first artery from the heart other than the coronary artery, is the final point of blood flow in retrograde perfusion. Therefore, air and debris in the heart are most likely to burst out during the time at which cardiac beating begins after the release of the aortic clamp, and the brachiocephalic artery becomes the boundary between retrograde perfusion and cardiac output. Then, the air and debris can flow to the brachiocephalic artery.

\section{Causes of SBI}

In this study, the all lesions were small and focal, therefore they were presented as grade A or B lesions. Based on the shape and size of the lesion, the etiology of SBI was interpreted as embolism due to microdebris and air by MRI image reading. Even SBI identified in the watershed region in 13 patients were believed to be caused by emboli because of the shape of the SBI in the watershed region. Knipp and colleague revealed that the cause of SBI after cardiac surgeries was embolism in their study, and the mechanism of embolism involves thromboemboli and air ${ }^{8,11}$. Oher researchers revealed that the cause of SBI after catheterization was air that was injected and debris in the ascending aorta ${ }^{13}$.

\section{Risk factor of SBI}

In the univariate analysis, age and aortic stenosis were considered the risk factors of SBI. Meanwhile, the multivariate analysis revealed that aortic stenosis was a risk factor. In aortic valve replacement for aortic valve stenosis, the possible cause of embolic lesions is the presence of debris generated when resecting aortic valves, and these debris may be responsible for brain infarcts, including emboli, after releasing the aortic clamp.

\section{Study limitations}

The current study had several limitations. First, this study was a non-randomized prospective study with no control group. Second, the timing of occurrence of SBI was not confirmed. MRI was performed on 
postoperative day 5, thus, we can only tell SBI occurred at some point from mid-surgery or postoperative day 5 . Some SBI might occurred postoperatively, not during the surgery.

\section{Conclusions}

The cause of SBI is associated with embolisms due to air and microdebris and the risk factor of SBI is AS. This study shows the low incidence of SBI after MICS. Thus, retrograde perfusion via the femoral artery may not increase incidence of SBI in selected MICS patients based on preoperative CT scan findings.

\section{Author contributions}

First Author: Shuhei Nishijima, $\mathrm{MD}^{1}$

Corresponding Author; Yoshitugu Nakamura, $\mathrm{MD}, \mathrm{PhD}^{1} *$

Data collection: Daiki Yoshiyama, $\mathrm{MD}^{1}$, Yuto Yasumoto, $\mathrm{MD}^{1}$, Miho Kuroda, MD ${ }^{1}$, , Taisuke Nakayama, $\mathrm{MD}^{1}$, Ryo Turuta, $\mathrm{MD}^{1}$, Yujiro Ito, $\mathrm{MD}^{1}$,

Statics: Fumiaki Shikata, MD, $\mathrm{PhD}^{1}$,

Data Analysis: Tetuji Takeda, $\mathrm{MD}^{2}$, Nobuyuki Katou, $\mathrm{MD}^{3}$

${ }^{1}$ Department of Cardiovascular Surgery, Chibanishi General Hospital, Matsudo, Chiba, Japan

${ }^{2}$ Department of Neuro Surgery, Chibanishi General Hospital, Matsudo, Chiba, Japan

${ }^{3}$ Department of Radiology, Chibanishi General Hospital, Matsudo, Chiba, Japan

Disclosures: The authors declare that there are no conflicts of interest.

\section{References}

1. Cheng DC, Martin J, Lal A, et al. Minimally invasive versus conventional open mitral valve surgery: a meta-analysis and systematic review. Innovations (Phila) 2011;6(2):84-103.

2. Dieberg G, Smart NA, King N. Minimally invasive cardiac surgery: A systematic review and meta-analysis. Int J Cardiol 2016;223:554-560.

3. Nishi H, Miyata H, Motomura N, et al. Which patients are candidates for minimally invasive mitral valve surgery? - Establishment of risk calculators using national clinical database. Circ J 2019;83(8):1674-1681.

4. Masuda T, Nakamura Y, Ito Y, et al. The learning curve of minimally invasive aortic valve replacement for aortic valve stenosis. Gen Thorac Cardiovas Surg 2019:10.1007/s11748-11019-01234-z.

5. Sun X, Lindsay J, Monsein LH, Hill PC, Corso PJ. Silent brain injury after cardiac surgery: a review: cognitive dysfunction and magnetic resonance imaging diffusion-weighted imaging findings. J Am Coll Cardiol 2012;60(9):791-797.

6. Floyd TF, Shah PN, Price CC, et al. Clinically silent cerebral ischemic events after cardiac surgery: their incidence, regional vascular occurrence, and procedural dependence. Ann Thorac Surg 2006;81(6):2160-2166.

7. Goksuluk H, Gulec S, Ozyuncu N, et al. Comparison of frequency of silent cerebral infarction after coronary angiography and stenting with transradial versus transfemoral approaches. Am J Cardiol 2018;122(4):548-553.

8. Knipp SC, Matatko N, Wilhelm H, et al. Evaluation of brain injury after coronary artery bypass grafting. A prospective study using neuropsychological assessment and diffusion-weighted magnetic resonance imaging. Eur J Cardiothorac Surg 2004;25(5):791-800.

9. Vermeer SE, Prins ND, den Heijer T, Hofman A, Koudstaal PJ, Breteler MM. Silent brain infarcts and the risk of dementia and cognitive decline. N Engl J Med 2003;348(13):1215-1222. 
10. Indja B, Woldendorp K, Vallely MP, Grieve SM. Silent brain infarcts following cardiac procedures: a systematic review and meta-analysis. J Am Heart Assoc 2019;8(9):e010920-e010920.

11. Knipp SC, Matatko N, Schlamann M, et al. Small ischemic brain lesions after cardiac valve replacement detected by diffusion-weighted magnetic resonance imaging: relation to neurocognitive function. Eur J Cardiothorac Surg 2005;28(1):88-96.

12. Friday G, Sutter F, Curtin A, et al. Brain magnetic resonance imaging abnormalities following off-pump cardiac surgery. Heart Surg Forum 2005;8(2):E105-109.

13. Omran H, Schmidt H, Hackenbroch M, et al. Silent and apparent cerebral embolism after retrograde catheterisation of the aortic valve in valvular stenosis: a prospective, randomised study. Lancet (London, England) 2003;361(9365):1241-1246.

13. Kim MJ, Lim YC, Oh SY, Kim BM, Kim BS, Shin YS. Thromboembolic events associated with electrolytic detachment of guglielmi detachable coils and target coils: comparison with use of diffusion-weighted MR imaging. J Korean Neurosurg Soc 2013;54(1):19-24.

15. Murai M, Hazui H, Sugie A, et al. Asymptomatic acute ischemic stroke after primary percutaneous coronary intervention in patients with acute coronary syndrome might be caused mainly by manipulating catheters or devices in the ascending aorta, regardless of the approach to the coronary artery. Circ J 2008;72(1):51-55.

16. Barbut D, Grassineau D, Lis E, Heier L, Hartman GS, Isom OW. Posterior distribution of infarcts in strokes related to cardiac operations. Ann Thorac Surg 1998;65(6):1656-1659.

\section{Figures legends}

Figure 1

Flowchart of patient selection for analyses.

Figure 2

Categorization of lesion detected on DWI. DWI spots measuring $<10 \mathrm{~mm}$ (A ). DWI spots measuring $>3$ $\mathrm{mm}(\mathbf{B})$. Single DWI lesion measuring $>10 \mathrm{~mm}(\mathbf{C})$. White arrows represent DWI lesions. 


\begin{tabular}{|c|c|c|c|c|}
\hline $\begin{array}{c}\text { Demographic } \\
\text { characteristics } \\
\text { of the } \\
\text { participants }\end{array}$ & Total & Without SCI & SCI & $P$ value \\
\hline & Patients $(n=174)$ & Patients $(n=148)$ & Patients $(n=26)$ & \\
\hline Age, years & $65.0 \pm 14.2$ & $64.1 \pm 14.6$ & $70.0 \pm 9.96$ & 0.007 \\
\hline Male & $92(52.9)$ & $81(54.7)$ & $11(42.31)$ & 0.289 \\
\hline $\begin{array}{c}\text { Body surface } \\
\text { area, } \mathrm{m}^{2}\end{array}$ & $1.57 \pm 0.21$ & $1.57 \pm 0.22$ & $1.55 \pm 0.18$ & 0.319 \\
\hline Hypertension & $90(51.7)$ & $72(48.7)$ & $18(66.67)$ & 0.0584 \\
\hline $\begin{array}{r}\text { Diabetes } \\
\text { mellitus }\end{array}$ & $19(10.9)$ & $15(10.1)$ & $4(14.8)$ & 0.492 \\
\hline CKD & $14(8.1)$ & $13(8.8)$ & $1(3.7)$ & 0.697 \\
\hline Hemodialysis & $1(0.57)$ & $1(0.68)$ & $0(0)$ & 1 \\
\hline COPD & $6(3.45)$ & $4(2.7)$ & $2(7.41)$ & 0.22 \\
\hline $\begin{array}{c}\text { History of } \\
\text { chronic arterial } \\
\text { fibrillation }\end{array}$ & $22(12.6)$ & $5(3.38)$ & $2(7.69)$ & 0.536 \\
\hline $\begin{array}{c}\text { History of } \\
\text { paroxysmal } \\
\text { atrial } \\
\text { fibrillation }\end{array}$ & $5(2.87)$ & $20(13.5)$ & $0(0)$ & 1 \\
\hline $\begin{array}{l}\text { History } \\
\text { cerebrovascula } \\
\text { r disease }\end{array}$ & $7(4.02)$ & $5(3.38)$ & $2(7.69)$ & 0.281 \\
\hline $\begin{array}{l}\text { Euro II } \\
\text { SCORE }\end{array}$ & $1.70 \pm 2.00$ & $1.71 \pm 2.06$ & $1.67 \pm 1.59$ & 0.449 \\
\hline AS (n) & $38(21.84)$ & $27(18.24)$ & $11(42.31)$ & 0.00995 \\
\hline AR (n) & $27(15.52)$ & $22(14.86)$ & $5(19.2)$ & 0.562 \\
\hline $\operatorname{MR}( \pm T R, n)$ & $87(50.00)$ & $78(52.70)$ & $9(34.62)$ & 0.136 \\
\hline
\end{tabular}

Table 1. Preoperative characteristics of 174 patients who underwent MICS and postoperative MRI, which were stratified according to silent brain infarction.

The values were presented as $\mathrm{n}(\%)$ or median (interquartile range).

MICS, minimally invasive cardiac surgery; MRI, magnetic resonance imaging 


\begin{tabular}{lc}
\hline & Patients $(\mathrm{n}=174)$ \\
\hline Isolated aortic valve replacement & $66(37.9)$ \\
Isolated mitral valve surgery & $77(44.2)$ \\
isolated mitral valve repairs & $69(39.6)$ \\
mitral valve replacements & $8(4.6)$ \\
Double valves & $20(11.5)$ \\
aortic/mitral valve & $4(2.3)$ \\
mitral/tricuspid valve & $16(9.2)$ \\
Other cardiac surgeries & $11(6.3)$ \\
Procedure time, minutes & $215( \pm 49.6)$ \\
Aortic cross-clamping time, minutes & $102.9( \pm 37.5)$ \\
Cardiopulmonary bypass time, minutes & $132.7( \pm 38.6)$ \\
Ventilation time, hours & $8.8( \pm 5.4)$ \\
Intensive care unit stay, hours & $57.6( \pm 26.4)$ \\
\hline
\end{tabular}

Table 2. Operative details of 174 patients who underwent MICS and postoperative MRI.

The values were presented as $\mathrm{n}(\%)$ or median (interquartile range).

MICS, minimally invasive cardiac surgery; MRI, magnetic resonance imaging 


\begin{tabular}{lc}
\hline Outcomes & Patients $(\mathrm{n}=174)$ \\
\hline Thirty-day mortality & 0 \\
Symptomatic stroke & 0 \\
Re-exploration & $2(1.1)$ \\
Renal failure & $5(2.9)$ \\
Reintubation & $1(0.5)$ \\
Surgical sight infection & $1(0.5)$ \\
Sepsis & 0 \\
Pneumonia & 0 \\
Aortic dissection & 0 \\
Femoral artery trauma & 0 \\
Postoperative atrial fibrillation & $25(14.3)$ \\
Lower extremity compartment syndrome & 0 \\
Intensive care unit readmission & 0 \\
Chest tube output (total or within 24 hours), $\mathrm{mL}$ & $171( \pm 165)$ \\
Timing of chest tube removal (day) & $1.6( \pm 1.0)$ \\
Blood tranfusion & $34( \pm 19.4)$ \\
Length of hospital stay, days & $9.4( \pm 7.5)$ \\
\hline
\end{tabular}

Table 3. Postoperative outcome of 174 patients who underwent MICS and postoperative MRI.

The values were presented as $\mathrm{n}(\%)$ or median (interquartile range)

MICS, minimally invasive cardiac surgery; MRI, magnetic resonance imaging 


\begin{tabular}{lc}
\hline Procedures associated with SBI & $\mathrm{n}=26(14.8)$ \\
\hline Isolated aortic valve surgery & $16(61.5)$ \\
Isolated mitral valve surgery & $7(26.9)$ \\
mitral valve repairs & $5(19.2)$ \\
mitral valve replacements & $2(7.7)$ \\
Double valve surgery & $2(7.7)$ \\
aortic and mitral valve & $0(0)$ \\
mitral and tricuspid valve & $2(7.7)$ \\
Other cardiac procedures (ASD/tumor) & $1(3.8)$ \\
\hline
\end{tabular}

Table 4. The number and percentage of silent brain infarction in each procedure.

The values were expressed as number and percentage (\%), unless

otherwise specified.

SBI, silent brain infarction; ASD, atrial septal defect 


\begin{tabular}{lrrrr}
\hline & \multicolumn{2}{c}{ Hemisphere } & \multicolumn{2}{c}{ Total } \\
\cline { 2 - 5 } Vascular region & Right & Left & & 1 \\
\hline ACA & 1 & 0 & 12 \\
MCA & 8 & 4 & 21 \\
PCA & 13 & 8 & 13 \\
Watershed & 6 & 7 & 1 \\
AICA & 0 & 1 & 13 \\
PICA & 6 & 7 & \\
Total & $34(56 \%)$ & $27(44 \%)$ & \\
\hline
\end{tabular}

Table 5. Occurrence of the lesion according to vascular region.

ACA, anterior cerebral artery; MCA, middle cerebral artery; PCA, posterior cerebral artery;

AICA, anterior inferior cerebral artery; PICA, posterior inferior cerebral artery 


\begin{tabular}{|l|c|c|c|c|}
\hline Variables & Odds ratio & $95 \% \mathrm{CI}$ & Wald & P values \\
\hline AVR for AS & 2.668 & $1.054-6.754$ & 4.286 & 0.038 \\
\hline Hypertension & 1.904 & $0.781-4.643$ & 2.004 & 0.157 \\
\hline $\begin{array}{l}\text { Age over 70 } \\
\text { years }\end{array}$ & 2.184 & $0.876-5.446$ & 2.808 & 0.094 \\
\hline
\end{tabular}

Table 6. Risk factors for postoperative silent cerebral infraction after minimally invasive cardiac surgery in the multivariate logistic regression model.

$\mathrm{AVR}$, aortic valve replacement; $\mathrm{AS}$, aortic stenosis; $\mathrm{Cl}$, confidence interval.

From July 2014 to July 2018 273 patients were underwent MICS and postoperative MRI

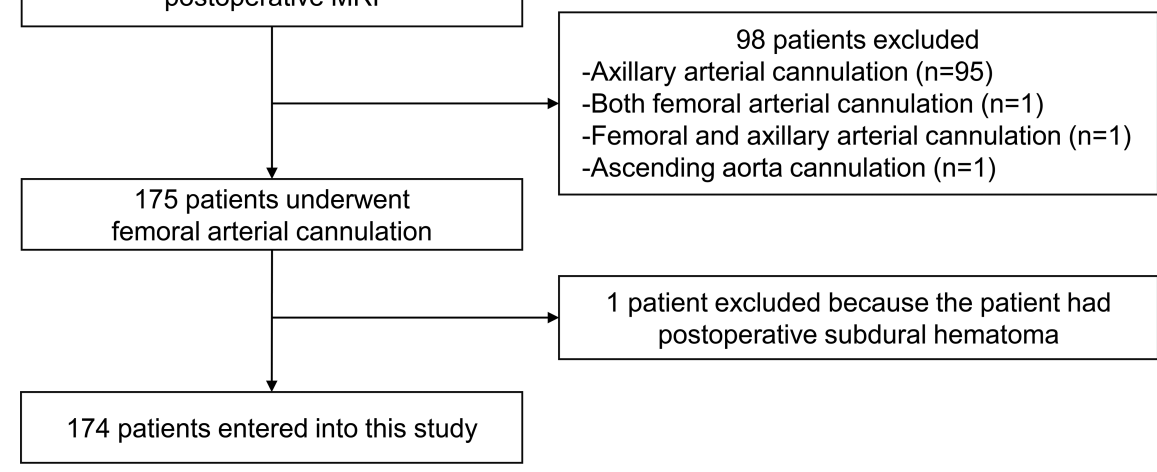




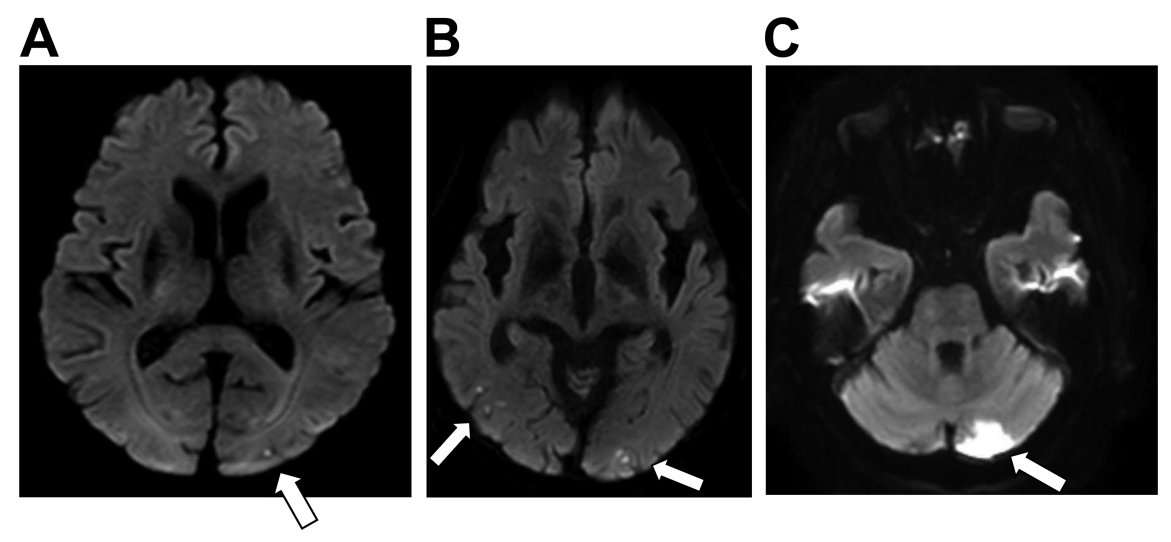

